\title{
Morphological predictors for lymph node metastases on computed tomography in colon cancer
}

\author{
Erik Rollvén ${ }^{1} \cdot$ Lennart Blomqvist $^{1} \cdot$ Emma Öistämö $^{2} \cdot$ Fredrik Hjern $^{3} \cdot$ György Csanaky ${ }^{4} \cdot$ Mirna Abraham-Nordling $^{5}$
}

Published online: 14 February 2019

(c) The Author(s) 2019

\begin{abstract}
Introduction/Background The aim of the study was to assess morphological predictors for lymph node metastases (Stage III disease) in colon cancer on computed tomography.

Methods and materials Ninety-four patients with histology-proven colon cancer (adenocarcinoma) who underwent elective primary curative resection between the years 2012 and 2014 were included. Contrast-enhanced CT examinations were independently reviewed by two blinded observers regarding tumor location, depth of tumor invasion, and presence of lymph node metastases. Ocular presence of internal heterogeneity and presence of irregular outer border were used as morphological criteria for lymph node involvement. Protocol-based histopathology after curative surgery served as reference standard. Sensitivity, specificity, positive (PPV) and negative (NPV) predictive values, and accuracy for each morphological criterion for prediction of stage III disease were calculated. Inter-observer agreement was compared using Kappa statistics.

Results According to histopathology, 59 patients were staged as I-II disease and 35 patients were staged as stage III disease. The presence of internal heterogeneity in a lymph node on CT resulted in moderate sensitivity (66-77\%) but high specificity (95-95\%) for prediction of Stage III disease by both observers. The presence of irregular outer border also resulted in poor sensitivity (49-54\%) but high specificity (97-97\%). The combination of either internal heterogeneity and/or irregular outer border per patient resulted in a moderate sensitivity (67-77\%) and high specificity (95-95\%), PPV (89-96\%), and NPV (84-88\%). Inter-observer agreement (Cohens Kappa) was 0.72. Consensus reading for the combined criteria resulted in sensitivity and specificity of $69 \%$ and $100 \%$, respectively.

Conclusion Using morphological criteria for lymph node metastases on CT examination in patients with colon cancer results in high specificity but moderate sensitivity in predicting stage III disease.
\end{abstract}

Keywords Colon cancer $\cdot$ Computed tomography $\cdot$ Staging $\cdot$ Stage III $\cdot$ Lymph nodes

\author{
Erik Rollvén \\ erik.rollven@ki.se \\ Lennart Blomqvist \\ lennart.k.blomqvist@ki.se \\ Emma Öistämö \\ emma.oistamo@dll.se \\ Fredrik Hjern \\ fredrik.hjern@ki.se \\ György Csanaky \\ gyocsa@ous-hf.no \\ Mirna Abraham-Nordling \\ mirna.abraham.nordling@ki.se
}

1 Department of Molecular Medicine and Surgery Karolinska Institutet, Department of Radiology, Karolinska University Hospital, Solna, 17176 Stockholm, Sweden

2 Department of Clinical Sciences, Karolinska Institutet, Stockholm, Sweden

3 Department of Clinical Sciences, Karolinska Institutet, Division of Surgery, Danderyd Hospital, Stockholm, Sweden

4 Department of Pathology and Clinical Cytology, Karolinska University Hospital, Stockholm, Sweden

5 Department of Molecular Medicine and Surgery Karolinska Institutet, Center for Digestive Diseases, Karolinska University Hospital, Stockholm, Sweden 


\section{Introduction}

Colorectal cancer is the most common malignancy in the gastrointestinal tract. In Sweden, the incidence is increasing with an aging population, while the mortality is slowly decreasing [1]. About two-thirds of colorectal tumors are located in the colon and one-third in the rectum.

The only curative treatment is surgical removal of the tumor containing the segment of the bowel together with its mesentery comprising local and regional lymph nodes. Adjuvant chemotherapy is recommended for patients with stage III disease (positive lymph node/s) and in some patients with stage II disease depending on the presence of additional histological risk factors after histopathological examination [2].

In colon cancer, a complete preoperative evaluation includes staging of the primary tumor and assessment of distant metastases in the liver and lungs with computed tomography (CT).

In recent years, some studies advocate and support the use of CT also for local staging of colon cancer containing treatment planning and selection of patients for neoadjuvant treatment.

In the Foxtrot trial, the selection was based on tumor stage, locally advanced tumor, with extramural tumor extensions, initially over $5 \mathrm{~mm}$ (ctT3c) but later $1 \mathrm{~mm}$ (ctT3a), and showed promising results in down-sizing the tumor stage with preoperative chemotherapy [3]. To select patients with colon cancer for neoadjuvant treatment, knowledge of prognostic factors including regional lymph node involvement will be even more important.

To date, there are no validated imaging criteria for the assessment of lymph node metastases in colon cancer. Previous studies have applied different criteria based on either size and/or morphology. Lymph node size $>1 \mathrm{~cm}$, short-long axis diameter ratio, internal heterogeneity (IH), irregular outer border (IOB), attenuation values $>100$ Hounsfield units (HU), and cluster of three or more normal sized lymph nodes, or any combination of the above, have all been used as a single or combined criteria [4-10].

A recently published study showed that assessment of morphology in the lymph nodes assessed by a single investigator, the combination of the criteria IH and IOB performed best in predicting nodal involvement, resulting in a sensitivity and specificity of $85 \%$ and $75 \%$, respectively [11].

The aim of this two observer study was to assess the accuracy and inter-observer variation for exclusively morphological criteria IH and IOB of lymph nodes on preoperative $\mathrm{CT}$, either alone or in combination for prediction of stage III colon cancer disease (Fig. 1).

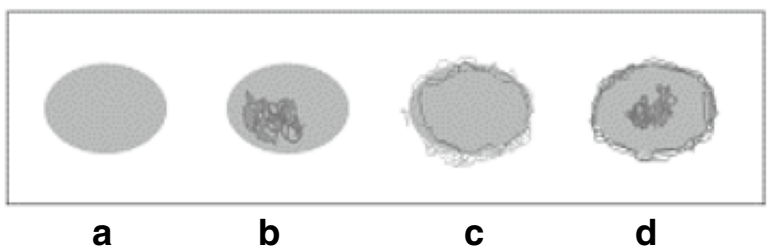

Note: $\mathrm{IH}=$ Internal Heterogeneity, $\mathrm{IOB}=$ Irregular Outer Border.

Fig. 1 a-d Schematic description of different lymph node appearances. a Normal lymph node appearance with homogenous internal architecture and well-defined outer border. b Lymph node with partial internal heterogeneity, IH. c Lymph node with circumferential irregular outer border, IOB. d Lymph node with IH and IOB within the same lymph node. $I H$ internal heterogeneity, $I O B$ Irregular outer border

\section{Methods and materials}

\section{Patients}

The study was performed at a University hospital in Stockholm, Sweden. Prior to initiation of the study, a fixed examination protocol for $\mathrm{CT}$, dedicated for reporting surgical and histopathological findings, had been established.

The inclusion criteria were that all patients, scheduled for surgery for histopathology-proven colon cancer (adenocarcinoma) from February 2012 to December 2014, were enrolled in the study. Informed consent was obtained from each patient before entering the study. The study was approved by the local ethics committee.

Patients were routinely scheduled for $\mathrm{CT}$ for screening of metastatic disease and assessment of the primary tumor. Demographic data, pre- and postoperative variables, carcinoembryonic antigen assay (CEA) $(\mathrm{ref}<5 \mu \mathrm{g} / \mathrm{l})$, and tumor location were recorded and are summarized in Table 1. In total, 112 consecutive patients were enrolled in the study.

Eighteen patients were excluded due to CT examination not fulfilling the standards stated in the study protocol (no intravenous contrast or different $\mathrm{CT}$ scanner) or presence of metastatic disease.

The remaining cohort $(n=94)$ comprised 45 women and 49 men with a median age of 72 (range 45-90) years and none had received any pre-treatment.

\section{Computed tomography}

All CT examinations included abdomen/pelvis and thorax with intravenous contrast $(300 \mathrm{mg} \mathrm{I} / \mathrm{ml}$, Iomeron, Bracco, $<60 \mathrm{~kg}$ body weight $120 \mathrm{ml},>60 \mathrm{~kg}$ body weight $150 \mathrm{ml}$ ) in portal-venous phase (delay $90 \mathrm{~s}$ after injection) using a 64-channel multislice CT scanner (GE Light 
Table 1 Demographics table of 94 patients/tumors

\begin{tabular}{|c|c|}
\hline Characteristics & Number $(\%)$ \\
\hline Sex (female/male) & $45 / 49$ \\
\hline Age (median, range) & $72(45-90)$ \\
\hline \multicolumn{2}{|l|}{ Histopathological evaluation } \\
\hline \multicolumn{2}{|l|}{ Tumor localization } \\
\hline Cecum & $25(27 \%)$ \\
\hline Ascending colon & $31(33 \%)$ \\
\hline Hepatic flexure & $1(1 \%)$ \\
\hline Transverse colon & $7(7 \%)$ \\
\hline Splenic flexure & $1(1 \%)$ \\
\hline Descending colon & $2(2 \%)$ \\
\hline Sigmoid colon & $27(29 \%)$ \\
\hline \multicolumn{2}{|l|}{ Tumor stage } \\
\hline $\mathrm{T} 1$ & $7(7 \%)$ \\
\hline $\mathrm{T} 2$ & $19(20 \%)$ \\
\hline T3 & $58(62 \%)$ \\
\hline $\mathrm{T} 4$ & $10(11 \%)$ \\
\hline \multicolumn{2}{|l|}{ Lymph node status } \\
\hline N0 & $59(63 \%)$ \\
\hline N1 & $19(20 \%)$ \\
\hline $\mathrm{N} 2$ & $16(17 \%)$ \\
\hline \multicolumn{2}{|l|}{ Positive lymph node status } \\
\hline T1 tumors & $0 / 7(0 \%)$ \\
\hline $\mathrm{T} 2$ tumors & $4 / 19(21 \%)$ \\
\hline T3 tumors & $25 / 58(43 \%)$ \\
\hline T4 tumors & $7 / 10(70 \%)$ \\
\hline \multicolumn{2}{|l|}{ Stage } \\
\hline Stage I & $22(23 \%)$ \\
\hline Stage II & $37(39 \%)$ \\
\hline Stage III & $35(37 \%)$ \\
\hline \multicolumn{2}{|l|}{ Lymph nodes, total number } \\
\hline Harvested lymph nodes PAD & 2086 \\
\hline Positive lymph nodes PAD total & 173 \\
\hline Positive lymph nodes PAD $<5 \mathrm{~mm}$ & 56 \\
\hline Positive lymph nodes PAD 5-10 mm & 62 \\
\hline Positive lymph nodes PAD > $10 \mathrm{~mm}$ & 18 \\
\hline
\end{tabular}

Speed-VCT). No oral contrast or bowel preparation was used. All examinations were performed at $120 \mathrm{kV}$ and with automatic current modulation. Median CTDI vol $(32 \mathrm{~cm})$ was $9.98 \mathrm{mGy}$ (range 5.27-19.46 mGy, SD 3.8). Original images were reconstructed to $5 \mathrm{~mm}$ slice thickness with 1-mm overlap (interval $4 \mathrm{~mm}$ ) and axial, coronal, and sagittal MPRs were routinely generated together with the original (thin slices) $0.625-\mathrm{mm}$ images.

\section{CT evaluation}

All CT examinations were retrospectively, independently reviewed by two GI radiologists with more than 20 years of experience in cross-sectional imaging of colorectal cancer (ER and LB) and blinded to all clinical information including tumor location. Examinations were assessed according to a dedicated evaluation proforma ("Appendix 1"). In case of no visible tumor in the colon, the tumor stage was assessed as T0. Both observers assessed tumor location (cecum, ascending colon, hepatic flexure, transverse colon, splenic flexure, descending colon, and sigmoid colon), tumor stage [T0, T1-T2, T3ab, T3cd, and T4 $(\mathrm{a}+\mathrm{b})]$. The tumor stage $\mathrm{T} 0$ was referred into the tumor stage $\mathrm{T} 1-\mathrm{T} 2$ group for further analysis.

The assessment of lymph node status ( $\mathrm{N} 0 / \mathrm{N}+)$ was limited to three morphological criteria in line with a previous study in assessment of lymph node metastases criteria in colon cancer with CT: (a) internal heterogeneity within at least one lymph node (IH) defined as mixed attenuation within the lymph node; (b) irregular outer border (IOB) defined as indistinct demarcation of the lymph node and; (c) combination of the two criteria, and therefore called combined criteria [11] (Fig. 2).

All measurements and assessments were performed on a Sectra Workstation IDS7 (version 15.1.14.41) both using the $5 \mathrm{~mm}$ reformatted images and the original thin slices $(0.625 \mathrm{~mm})$, the latter primary for detection of small lymph nodes $(\leq 5 \mathrm{~mm})$. The original thin slices were also in some cases, where a $5 \mathrm{~mm}$ thickness was considered too thick, additionally merged into 2 and $3 \mathrm{~mm}$ slice thickness.

\section{Surgery}

The median interval time between preoperative $\mathrm{CT}$ and the curative surgery was 22 (range 1-54) days. All patients in the study were operated in a curative elective setting and according to colorectal surgical praxis. The resection of colon cancer was made by the principle of clear lateral margins, resection of the loco-regional lymph node bearing mesentery. A dedicated protocol was used for reporting of surgical findings.

\section{Histopathology}

Histopathology was performed according to standard procedures at the university hospital pathology department by a specialized GI pathologist (TNM version 7). From the pathologists' original report, the $\mathrm{T}$ - and $\mathrm{N}$-stages, total number of harvested and metastatic lymph nodes served as reference standard. For the majority of the patients $(n=80$, $85 \%$ ), the harvested lymph nodes, both benign and metastatic lymph nodes, were categorized according to size ( $<5 \mathrm{~mm}, 5-10 \mathrm{~mm}$, and $>10 \mathrm{~mm})$. Tumor deposits (N1c) assessed by the pathologist were characterized and regarded as equivalent with lymph nodes when comparison with $\mathrm{CT}$ images (Appendix 2). 

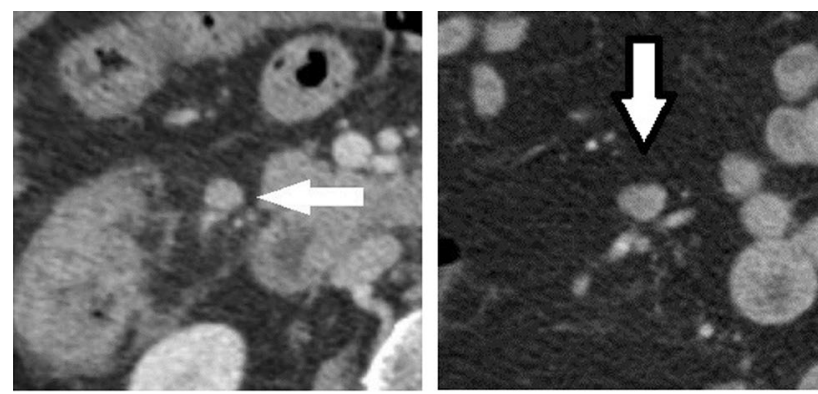

a

b

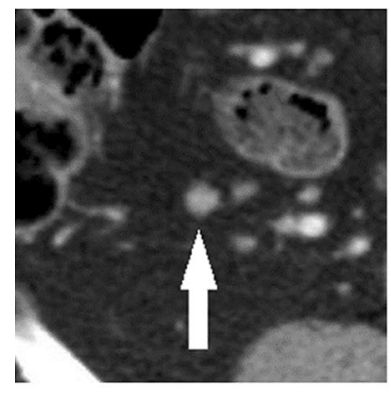

C

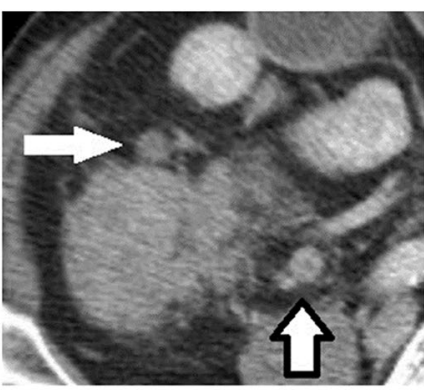

d

Note: $\mathrm{IH}=$ Internal Heterogeneity, $\mathrm{IOB}=$ Irregular Outer Border.

Fig. 2 a-d Examples of the different morphological CT criteria. a Patient with a pT3a, N0 tumor in the sigmoid colon. Assessed as N0 by both observers-not fulfilling the IH or IOB criteria. White arrow shows a $7 \times 6 \mathrm{~mm}$ normal lymph node. b Patient with a pT3a, N2 (4 lymph node metastases out of 34 lymph nodes) tumor in the cecum assessed as N2 and N1, respectively, by both observers by the criteria IH. White arrow shows a $9 \times 5 \mathrm{~mm}$ lymph node with IH. $\mathbf{c}$ Patient with a pT3c, N1 (2 lymph node metastases out of 25 lymph nodes) tumor in the sigmoid colon assessed as N1 by both observers by the criteria IOB. White arrow shows a $7 \times 6 \mathrm{~mm}$ lymph node with IOB. d Patient with a pT4b, N2 (16 lymph node metastases out of 29 lymph nodes) tumor in the cecum assessed as $\mathrm{N} 2$ by both observers by the criteria $\mathrm{IH}$. Black/white arrow shows a $6 \times 6 \mathrm{~mm}$ lymph node with $\mathrm{IH}$. White arrow shows a $7 \times 6 \mathrm{~mm}$ lymph node with $\mathrm{IH}$ and IOB within the same lymph node. $I H$ internal heterogeneity, $I O B$ irregular outer border

\section{Statistical analysis}

Descriptive statistics were applied to different tumor stages and lymph node characteristics calculating sensitivity, specificity, positive predictive value (PPV), and negative predictive value (NPV) for the prediction of stage III disease. Statistical significance was set to $p \leq 0.05$. Inter-observer variation was classified using Cohens Kappa statistics. Data were evaluated using the statistical analysis software, IBM SPSS Statistics (Version 24).

\section{Results}

\section{Histopathology}

The majority of the tumors, 56 out of $94(60 \%)$, were right sided and located in the cecum and ascending colon. The histopathological distribution of $\mathrm{T}$ - and $\mathrm{N}$-stages in the cohort is presented in Table 1. Thirty-five (37\%) patients were lymph node positive (Table 1).

A total of 2086 lymph nodes were harvested (in median 20 lymph nodes per patient, range 3-69), of which 173 lymph nodes were assessed as metastatic (in median 3 lymph nodes per patient, range 1-21 (Table 1).

\section{CT evaluation}

\section{T-stage}

T-stage assessed by CT is presented in Table 2. In 16 and 21 patients, respectively, the observers were not able to detect any tumor (ctT0). Twelve (75\%) and fourteen (67\%), respectively, of those patients had a pT1-T2 tumor according to histopathology. When stratifying ctT-stage, in not locally advanced (ctT1-T3ab) and locally advanced (ctT3 cd-T4), the sensitivity and specificity for observer 1 compared to the pT-stage were $79 \%$ and $96 \%$, and for observer $2,61 \%$ and $97 \%$ and consensus $75 \%$ and $97 \%$, respectively. Inter-observer agreement for ctT-stage (Cohens Kappa) was 0.76 (good agreement).

\section{$\mathrm{N}$-stage}

The number of lymph nodes and patients with each morphological feature assessed by CT is displayed in Table 3 as well as sensitivity, specificity, PPV, and NPV.

\section{Internal heterogeneity (IH)}

IH was detected in at least one lymph node in $28(30 \%)$ and $26(28 \%)$ out of 94 patients by observers 1 and 2, respectively. A total number of 93 and 64 lymph nodes, respectively, with this morphologic feature were detected for each observer. Sensitivity/specificity for ctN-stage compared to $\mathrm{pN}$-stage for both observers were $77 / 95 \%$ and $66 / 95 \%$, respectively. Inter-observer agreement (Cohens Kappa) for ctN-stage was 0.74 (good agreement).

\section{Irregular outer border (IOB)}

Lymph nodes assessed as presenting IOB were detected at least in one lymph node in 20 and 19 out of 94 patients, respectively, by observers 1 and 2. Sensitivity/specificity for $\mathrm{ctN}$-stage compared to $\mathrm{pN}$-stage for both observers were $54 / 97 \%$ and $49 / 97 \%$, respectively.

\section{Both internal heterogeneity and irregular outer border in one lymph node}

The combination of IH and IOB in the same lymph node was assessed in 18 out of 94 patients by both observers. 
Table 2 Predictive values for T-staging

\begin{tabular}{|c|c|c|c|c|c|c|}
\hline & \multicolumn{2}{|l|}{ Obs 1} & \multicolumn{2}{|l|}{ Obs 2} & \multicolumn{2}{|l|}{ Consensus } \\
\hline & $\begin{array}{l}\text { T0-T3ab } \\
(n=71)\end{array}$ & $\begin{array}{l}\mathrm{T} 3 \mathrm{~cd}-\mathrm{T} 4 \\
(n=23)\end{array}$ & $\begin{array}{l}\text { T0-T3ab } \\
(n=79)\end{array}$ & $\begin{array}{l}\mathrm{T} 3 \mathrm{~cd}-\mathrm{T} 4 \\
(n=15)\end{array}$ & $\begin{array}{l}\text { T0-T3ab } \\
(n=71)\end{array}$ & $\begin{array}{l}\text { T3cd- } \\
\text { T4 } \\
(n=23)\end{array}$ \\
\hline $\mathrm{TN}$ & 22 & 65 & 17 & 64 & 21 & 64 \\
\hline $\mathrm{FN}$ & 1 & 6 & 2 & 11 & 2 & 7 \\
\hline FP & 6 & 1 & 11 & 2 & 7 & 2 \\
\hline $\mathrm{TP}$ & 65 & 22 & 64 & 17 & 64 & 21 \\
\hline Sensitivity (\%) & 96 & 79 & 97 & 61 & 97 & 75 \\
\hline Specificity (\%) & 79 & 96 & 61 & 97 & 75 & 97 \\
\hline PPV (\%) & 79 & 96 & 85 & 90 & 90 & 91 \\
\hline NPV (\%) & 96 & 79 & 90 & 85 & 91 & 90 \\
\hline
\end{tabular}

$T N$ true negative, $F N$ false negative, $F P$ false positive, $T P$ true positive, $P P V$ positive predictive value, $N P V$ negative predictive value

\begin{tabular}{|c|c|c|c|c|c|c|c|c|}
\hline & \multicolumn{2}{|l|}{$\mathrm{IH}$} & \multicolumn{2}{|l|}{ IOB } & \multicolumn{2}{|c|}{ IH/IOB } & \multicolumn{2}{|c|}{ IH/IOB/(IH/IOB) } \\
\hline & Obs 1 & Obs 2 & Obs 1 & Obs 2 & Obs 1 & Obs 2 & Obs 1 & Obs 2 \\
\hline No of pat & 28 & 26 & 20 & 19 & 18 & 18 & 28 & 27 \\
\hline No of $\ln$ & 93 & 64 & 28 & 24 & 35 & 30 & 93 & 66 \\
\hline $\mathrm{TN}$ & 58 & 56 & 57 & 57 & 57 & 57 & 58 & 56 \\
\hline FN & 8 & 12 & 16 & 18 & 18 & 19 & 8 & 11 \\
\hline FP & 1 & 3 & 2 & 2 & 2 & 2 & 1 & 3 \\
\hline $\mathrm{TP}$ & 27 & 23 & 19 & 17 & 17 & 16 & 27 & 24 \\
\hline Sensitivity & 77 & 66 & 54 & 49 & 49 & 46 & 77 & 67 \\
\hline Specificity & 95 & 95 & 97 & 97 & 97 & 97 & 95 & 95 \\
\hline PPV & 96 & 88 & 91 & 90 & 90 & 89 & 96 & 89 \\
\hline NPV & 88 & 82 & 78 & 76 & 76 & 75 & 88 & 84 \\
\hline
\end{tabular}

$T N$ true negative, $F N$ false negative, $F P$ false positive, $T P$ true positive, $P P V$ positive predictive value, $N P V$ negative predictive value, $I H$ internal heterogeneity, $I O B$ irregular outer border, $I H / I O B /(I H / I O B) \mathrm{N}+$
Table 3 Sensitivity, specificity, positive and negative predictive values for different $\mathrm{CT}$ criteria for lymph node metastases
Sensitivity/specificity for $\mathrm{ctN}$-stage compared to $\mathrm{pN}$-stage for both observers were $49 / 97 \%$ and $46 / 97 \%$, respectively.

\section{Nodal stage -Either IH or IOB or IH/IOB}

Combining the three possible criteria, IH or IOB or IH/ IOB, for lymph nodes and if at least one event occurred in one patient, this was assessed in 28 and 27 out of 94 patients, respectively, by both observers. Sensitivity/specificity for $\mathrm{ctN}$-stage compared to $\mathrm{pN}$-stage for both observers were $77 / 95 \%$ and $67 / 95 \%$, respectively (Table 3 ). Interobserver agreement (Cohens Kappa) for ctN-stage was 0.72 (good agreement). Consensus ctN-stage compared to $\mathrm{pN}$-stage for metastases resulted in a sensitivity and specificity of $69 \%$ and $100 \%$.

In the present study, the major discrepancy was understaging (false negatives, FN) (Table 3). For observer 1, there were $8 \mathrm{FN}$ cases. In these patients, there were a total of sixteen metastatic lymph nodes according to histopathology and none of the metastatic nodes were $>10 \mathrm{~mm}$ in size. Eleven (69\%) metastatic lymph nodes were $<5 \mathrm{~mm}$. For observer 2 , there were $11 \mathrm{FN}$ cases with a total of 24 metastatic lymph nodes according to histopathology and none of the metastatic nodes $>10 \mathrm{~mm}$ in size. Fifteen (62\%) metastatic lymph nodes were $<5 \mathrm{~mm}$ according to histopathology.

In consensus reading, the observers had $11 \mathrm{FN}$ cases according to histopathology. Of the total number of 25 lymph node metastases, none were $>10 \mathrm{~mm}, 8(32 \%)$ lymph nodes were between 5 and $10 \mathrm{~mm}$, and 17 (68\%) were $<5 \mathrm{~mm}$ in size.

\section{Discussion}

The aim of this study was to evaluate the diagnostic predictive performance of morphological lymph node features on CT such as IH, IOB, and a combination of those predictive for stage III disease in colon cancer. 
The results of this study show that assessment based on $\mathrm{IH}$ alone and the combination of IH and IOB results in a moderate sensitivity but high specificity for prediction of stage III disease. The assessment of IH alone is the strongest predictor of the morphological criteria for metastases supporting the hypothesis of tumor invasion of a lymph node with regular outer border before the periglandular growth (IOB). These results are in line with other comparable studies, yet inferior to the work using morphological criteria in MRI of rectal cancer of Brown et al. where mixed signal intensity or irregular border resulted in a sensitivity of $85 \%$ and specificity of 97\% [12]. Furthermore, the results are better regarding specificity than reports in a recent metaanalysis, including 16 studies, wherein a sensitivity and specificity of CT for nodal involvement of $71 \%$ and $67 \%$ were reported [13]. The higher specificity suggests that analyzing lymph node with these criteria could predict patients not having stage III disease.

Furthermore, in histopathological examinations it has been shown that nearly $50 \%$ of the lymph nodes in colon cancer are below $5 \mathrm{~mm}$ in size $[14,15]$. This is also verified in the present study where within the $\mathrm{pN}+$ population in the present study, 56 out of 136 pathologically identified metastatic lymph nodes (41\%) were below $5 \mathrm{~mm}$ in size. On the other hand, no more than 18 out of 173 (10\%) metastatic lymph nodes (in 9 out of 35 patients) exceeded $10 \mathrm{~mm}$ in size. The large proportion of small compared to large lymph nodes illustrates the challenge for imaging in assessment of metastatic lymph nodes.

The use of automatic tube current modulation in the CT protocol helped keeping the noise level constant between images within examinations and between patients. With a constant image quality, a more optimal review can be performed. Volumetric high-resolution CT images used today allow a detailed assessment of size and morphology of pathological lesions. Yet, the signal-to-noise ratio is low when using thin slices $(0.625 \mathrm{~mm})$ which has to be considered when evaluating morphology to not confuse image noise with heterogeneity. For this reason, we used 5-mm sections for morphological assessment and thinner sections for detection of lymph nodes.

Regarding the T-stage, the classification of tumor stage, into not locally advanced (T1-T3ab) or locally advanced (T3cd-T4) resulted in moderate sensitivity (61-79\%) and high specificity (97-97\%). The results are in line with a recent meta-analysis including four studies with a pooled sensitivity of $77 \%$ and specificity of $70 \%$ [13]. The current study shows higher specificity due to low false-positive assessment (Table 3). Other reports using MRI for detection and staging of colon cancer have shown similar results [16-18]. According to histopathology, 28 out of 94 tumors in our study were of tumor stage T3 cd-T4, and out of those, 22 and 17 tumors were correctly classified by observers 1 and 2, respectively. The difference was mainly due to underestimation of extramural disease (EMD) in $\mathrm{T} 3 \mathrm{~cd}$ tumors. One patient with a T4a tumor, misclassified by both observers, illustrates challenges in identifying small serosa involvement.

In consensus reading, both observers classified two tumors as not visible (ctT0, ctN0), while they were classified as pT3a (ascending colon) and pT3b (cecum) according to histopathology and both patients were node positive (pN1 and pN2). The first patient had two lymph node metastases, $1<5 \mathrm{~mm}$ and one $5-10 \mathrm{~mm}$. In the other patient, there were four lymph node metastases, all below $5 \mathrm{~mm}$ in size. Since both readers were blinded of the tumor location, the assessment of lymph node in these ctT0 patients was therefore challenging.

In rectal cancer patients, the selection for neoadjuvant treatment is based on the clinical stage where magnetic resonance imaging plays the most important role. Wellknown important prognostic factors in colorectal cancer are tumor stage, extramural vascular invasion, and lymph node involvement [2]. Hence, neoadjuvant treatment has resulted in reduction in tumor size and recurrence rate [19]. For colon cancer, the ongoing FOxTROT trial uses only extramural tumor depth as inclusion criteria based on CT and reported a sensitivity and specificity for lymph node involvement to $83 \%$ and $44 \%$, respectively, and was therefore not used. The present study shows that four out of 19 (21\%) pT2 tumors had lymph node metastases (a total of four lymph nodes, $3<5 \mathrm{~mm}$ and one $5-10 \mathrm{~mm}$ in size) according to histopathology. Of those four tumors, two were correctly diagnosed as node positive by one observer and misclassified by the other observer. These four patients would potentially benefit from neoadjuvant treatment. In a previous trial using both CT and MRI regarding lymph node metastases in early colorectal cancer with submucosal invasion, a sensitivity of $79 \%$ and specificity of $75 \%$ were achieved when using size criteria for metastasis of $4.1 \mathrm{~mm}$ in short diameter of the lymph node. The authors point out to pay more attention to small nodes in early cancer because it is more likely to be malignant than reactive as in more advanced cancers [20].

Some studies using FDG PET/CT have reported high specificity but low sensitivity for nodal staging, suggesting that FDG PET/CT is of limited additional value in detecting regional lymph node metastases due to high false-negative rate [21, 22].

In metastatic colon cancer disease (liver, lung, or paraaortal nodes), some authors claim that FDG PET/CT may alter the management, while other authors claim that it does not [23, 24]. There is evidence supporting PET/ $\mathrm{CT}$ as a superior staging modality for patients with metastatic colorectal disease; current National Comprehensive Cancer Network guidelines for colorectal cancer do not 
recommend the use of FDG PET/CT in preoperative staging of these patients [25].

Currently, FDG PET/CT has found a role in the evaluation of patients prior to major surgery or with an unexplained rise in their CEA that is suspected to represent a tumor recurrence [26, 27].

According to the results in the current study, morphological CT criteria alone are not sufficient for nodal staging. The low sensitivity reflects limited presence of visible morphological features for metastases on CT imaging. The high specificity is caused by false negatives and absence of morphological CT criteria in positive lymph node patients according to histopathology. The explanation for this could be that small lymph nodes $(<5 \mathrm{~mm})$ and the microscopic tumor growth in normal lymph nodes are not detectable with CT. If selection of patients for neoadjuvant treatment was done using morphological criteria from this study (IH and IOB), eleven out of 35 patients in the cohort would potentially have been undertreated and none overtreated. On the other hand, if a certain neoadjuvant treatment has potential significant side effects to the extent that overtreatment is not justified, the high specificity of the morphological criteria on CT would be an advantage.

The strength of this study is the homogenous consecutive patient cohort, prospective data collection, all patients were examined with the same CT, an independent evaluation, and all patients having primary curative surgery without neoadjuvant treatment allowing protocol-based detailed histopathology of the resected specimen as a reference.

There are some limitations with this study: the assessment of the morphological image criteria was performed by two highly experienced GI radiologists and may not simulate the clinical everyday setting. The study was on a per-patient basis and no matching of individual lymph nodes between imaging and histopathology was performed.
Another limitation might be that software using quantitative analysis of morphology such as texture analysis was not used. Texture analysis may potentially have a role in the context of characterizing regional lymph nodes on CT in colon cancer although the approach in this setting is rather unexplored. However, small size of lymph nodes will probably then be one of the challenges when performing such analysis.

To conclude, morphological criteria for lymph node metastases on CT in colon cancer result in high specificity and moderate sensitivity in predicting stage III disease.

Author contributions Study concepts and design: ER, MAN, LB, GS, EÖ, FH. Performing study: ER, LB, MAN, EÖ, GS, FH. Evaluating exams: ER, LB, GS. Collection of data: ER, EÖ, MAN. Analysis of data: ER, MAN, LB. Manuscript preparation: ER, LB, MAN. Manuscript reviewing and editing: ER, MAN, LB, FH. Final approval of the version to be published: ER, MAN, LB, GS, EÖ, FH.

Funding Financial support was provided through the regional agreement on medical training and clinical research (ALF) between the Stockholm County Council and Karolinska Institutet and Bengt Ihre Foundation.

\section{Compliance with ethical standards}

Conflict of interest The authors declare that they have no competing interests.

Ethical approval The study was approved by the regional ethical review board (2011/1371-31/3).

Informed consent Each author has participated sufficiently in the submission and takes public responsibility for its content.

Open Access This article is distributed under the terms of the Creative Commons Attribution 4.0 International License (http://creativeco mmons.org/licenses/by/4.0/), which permits unrestricted use, distribution, and reproduction in any medium, provided you give appropriate credit to the original author(s) and the source, provide a link to the Creative Commons license, and indicate if changes were made. 


\section{Appendix 1}

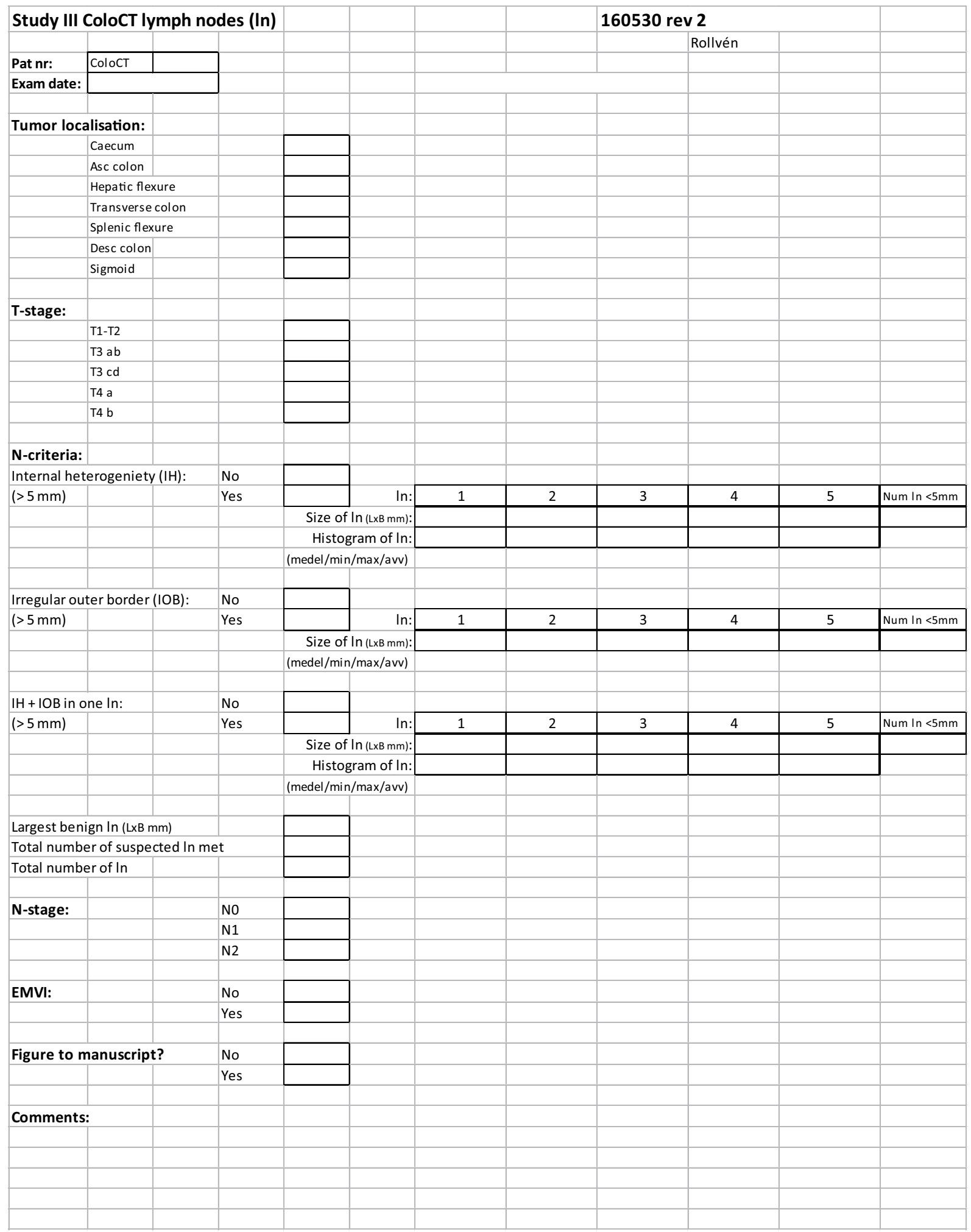




\section{Appendix 2}

\section{Patologprotokoll}
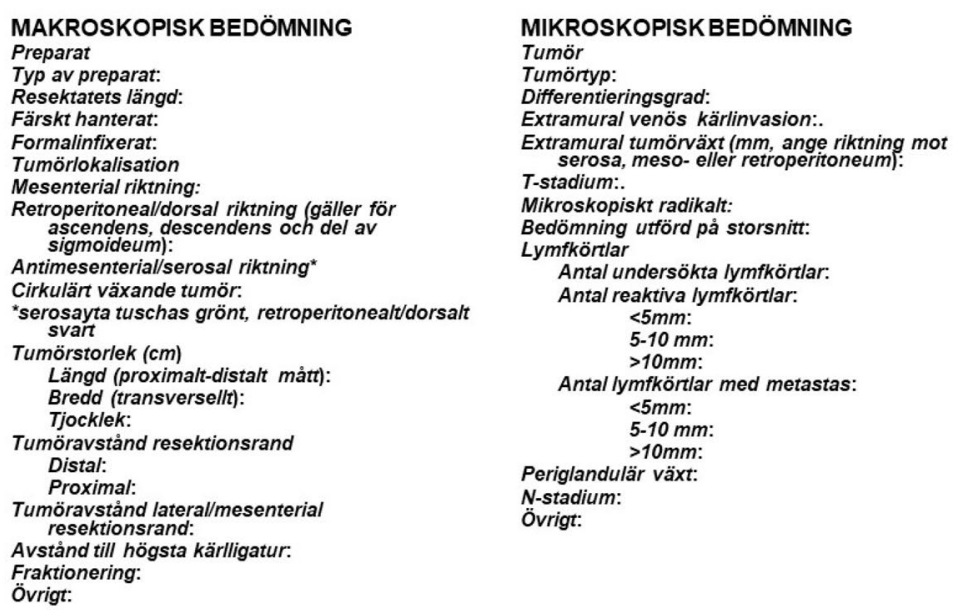

N-stadium:

Fraktionering

\section{References}

1. SCRCR. Colon cancer registry report 2011 Sweden. 2011.

2. Quasar Collaborative G, Gray R, Barnwell J, McConkey C, Hills RK, Williams NS, et al. Adjuvant chemotherapy versus observation in patients with colorectal cancer: a randomised study. Lancet. 2007;370(9604):2020-9.

3. Foxtrot Collaborative G. Feasibility of preoperative chemotherapy for locally advanced, operable colon cancer: the pilot phase of a randomised controlled trial. Lancet Oncol. 2012;13(11):1152-60.

4. Smith NJ, Barbachano Y, Norman AR, Swift RI, Abulafi AM, Brown G. Prognostic significance of magnetic resonance imagingdetected extramural vascular invasion in rectal cancer. Br J Surg. 2008;95(2):229-36.

5. Acunas B, Rozanes I, Acunas G, Celik L, Sayi I, Gokmen E. Preoperative CT staging of colon carcinoma (excluding the rectosigmoid region). Eur J Radiol. 1990;11(2):150-3.

6. Burton S, Brown G, Bees N, Norman A, Biedrzycki O, Arnaout A, et al. Accuracy of CT prediction of poor prognostic features in colonic cancer. The British journal of radiology. 2008;81(961):10-9.

7. Zbar AP, Rambarat C, Shenoy RK. Routine preoperative abdominal computed tomography in colon cancer: a utility study. Techniques in coloproctology. 2007;11(2):105-9.

8. Harvey CJ, Amin Z, Hare CM, Gillams AR, Novelli MR, Boulos PB, et al. Helical CT pneumocolon to assess colonic tumors: radiologic-pathologic correlation. AJR Am J Roentgenol. 1998;170(6):1439-43.
9. Gazelle GS, Gaa J, Saini S, Shellito P. Staging of colon carcinoma using water enema CT. Journal of computer assisted tomography. 1995;19(1):87-91.

10. Hundt W, Braunschweig R, Reiser M. Evaluation of spiral CT in staging of colon and rectum carcinoma. Eur Radiol. 1999;9(1):78-84.

11. Rollven E, Abraham-Nordling M, Holm T, Blomqvist L. Assessment and diagnostic accuracy of lymph node status to predict stage III colon cancer using computed tomography. Cancer Imaging. 2017;17(1):3.

12. Brown G, Richards CJ, Bourne MW, Newcombe RG, Radcliffe AG, Dallimore NS, et al. Morphologic predictors of lymph node status in rectal cancer with use of high-spatial-resolution MR imaging with histopathologic comparison. Radiology. 2003;227(2):371-7.

13. Nerad E, Lahaye MJ, Maas M, Nelemans P, Bakers FC, Beets GL, et al. Diagnostic Accuracy of CT for Local Staging of Colon Cancer: A Systematic Review and Meta-Analysis. AJR Am J Roentgenol. 2016;207(5):984-95.

14. Rodriguez-Bigas MA, Maamoun S, Weber TK, Penetrante RB, Blumenson LE, Petrelli NJ. Clinical significance of colorectal cancer: metastases in lymph nodes $<5 \mathrm{~mm}$ in size. Annals of surgical oncology. 1996;3(2):124-30.

15. Markl B, Rossle J, Arnholdt HM, Schaller T, Krammer I, Cacchi $\mathrm{C}$, et al. The clinical significance of lymph node size in colon cancer. Mod Pathol. 2012;25(10):1413-22.

16. Nerad E, Lambregts DM, Kersten EL, Maas M, Bakers FC, van den Bosch HC, et al. MRI for Local Staging of Colon Cancer: Can MRI Become the Optimal Staging Modality for Patients With Colon Cancer? Diseases of the colon and rectum. 2017;60(4):385-92. 
17. Rollven E, Holm T, Glimelius B, Lorinc E, Blomqvist L. Potentials of high resolution magnetic resonance imaging versus computed tomography for preoperative local staging of colon cancer. Acta Radiol. 2013;54(7):722-30.

18. Hunter C, Siddiqui M, Georgiou Delisle T, Blake H, Jeyadevan N, Abulafi M, et al. CT and 3-T MRI accurately identify T3c disease in colon cancer, which strongly predicts disease-free survival. Clinical radiology. 2017;72(4):307-15.

19. Erlandsson J, Holm T, Pettersson D, Berglund A, Cedermark B, Radu C, et al. Optimal fractionation of preoperative radiotherapy and timing to surgery for rectal cancer (Stockholm III): a multicentre, randomised, non-blinded, phase 3, non-inferiority trial. Lancet Oncol. 2017;18(3):336-46.

20. Choi J, Oh SN, Yeo DM, Kang WK, Jung CK, Kim SW, et al. Computed tomography and magnetic resonance imaging evaluation of lymph node metastasis in early colorectal cancer. World $\mathbf{J}$ Gastroenterol. 2015;21(2):556-62.

21. Abdel-Nabi H, Doerr RJ, Lamonica DM, Cronin VR, Galantowicz PJ, Carbone GM, et al. Staging of primary colorectal carcinomas with fluorine-18 fluorodeoxyglucose whole-body PET: correlation with histopathologic and CT findings. Radiology. 1998;206(3):755-60.

22. Furukawa H, Ikuma H, Seki A, Yokoe K, Yuen S, Aramaki $\mathrm{T}$, et al. Positron emission tomography scanning is not superior to whole body multidetector helical computed tomography in the preoperative staging of colorectal cancer. Gut. 2006;55(7):1007-11.

23. Lee JH, Lee MR. Positron emission tomography/computed tomography in the staging of colon cancer. Annals of coloproctology. 2014;30(1):23-7.

24. Cipe G, Ergul N, Hasbahceci M, Firat D, Bozkurt S, Memmi $\mathrm{N}$, et al. Routine use of positron-emission tomography/computed tomography for staging of primary colorectal cancer: does it affect clinical management? World journal of surgical oncology. 2013;11:49.

25. Benson AB, 3rd, Venook AP, Cederquist L, Chan E, Chen YJ, Cooper HS, et al. Colon Cancer, Version 1.2017, NCCN Clinical Practice Guidelines in Oncology. J Natl Compr Canc Netw. 2017;15(3):370-98.

26. Expert Panel on Gastrointestinal I, Fowler KJ, Kaur H, Cash BD, Feig BW, Gage KL, et al. ACR Appropriateness Criteria((R)) Pretreatment Staging of Colorectal Cancer. J Am Coll Radiol. 2017;14(5S):S234-S44.

27. Briggs RH, Chowdhury FU, Lodge JP, Scarsbrook AF. Clinical impact of FDG PET-CT in patients with potentially operable metastatic colorectal cancer. Clinical radiology. 2011;66(12):1167-74.

Publisher's Note Springer Nature remains neutral with regard to jurisdictional claims in published maps and institutional affiliations. 